\title{
Non-linear Identification Applied to Broadband Turbomachinery Noise
}

\author{
Gareth J. Bennett*, Ian Davis ${ }^{\dagger}$ \\ Trinity College Dublin, Ireland. \\ Ulf Tapken $\ddagger$ \\ German Aerospace Center (DLR), Institute of Propulsion Technology, Engine Acoustics Branch \\ John Mahon ${ }^{\S}$ \\ Trinity College Dublin, Ireland.
}

\begin{abstract}
Coherence based source analysis techniques can be used to identify the contribution of broadband noise sources in the exhaust of a gas turbine and hence enable the design of noise reduction devices. However, when the broadband noise source propagates in a non-linear fashion the identified contribution using ordinary coherence methods will be inaccurate. In this paper, an analysis technique to enable the contribution of linear and non-linear mechanisms to the propagated sound to be identified is reported. An bench-test experimental rig to study the propagation of noise through a rotor/stator set-up using a vane-axial fan mounted in a duct so that non-linear interactions between a sound source and the fan could be investigated is described. The technique which is used to identify non-linear noise contributions generated by the interaction of the rotor and propagated narrowband noise is reported and validated using this rig. The techniques are subsequently applied to data generated from within an FP7 Collaborative European Aeroacoustics Project: TEENI. The analysis techniques enabled non-linear interactions to be identified and linear and non-linear contributions to be determined.
\end{abstract}

\section{Introduction}

The reduction of the principal sources of aero-engine noise; the jet and the tonal contributions from the fan, compressor and turbine, has resulted in a new noise floor being reached. This noise floor will limit the benefits to be gained by further reducing these dominant components, unless the noise sources which set this threshold are in turn reduced. Of these, band-limited combustion noise and the broadband core noise sources of turbomachinery are significant contributors. The current trend towards high-bypass engines (which tend to reduce jet noise) and low Nox combustors (which may modify the noise characteristics) will result in broadband core noise becoming a more significant source.

Coherence-based noise source identification techniques can be used to identify the contribution of combustion noise to near and far field acoustic measurements of aero-engines. Karchmer and Reshotko ${ }^{1,2}$ and Reshotko and Karchmer ${ }^{3}$ used the ordinary coherence function between internal measurements and farfield microphones and derived the core noise at farfield locations by calculating the coherent output power (COP): a technique reported initially by Halvorsen and Bendat ${ }^{4}$. Karchmer ${ }^{5}$ also used the conditioned coherency function to determine where the source region for core noise was located. Extraneous noise contamination at an internal microphone location can result in the derived core noise at the farfield location being significantly lower than the true value. For such situations, Shivashankara ${ }^{6,7}$ used Chung's ${ }^{8}$ flow noise rejection technique, to identify the internal core noise contribution to farfield noise measurements. Hsu and Ahuja ${ }^{9}$ extended

*Lecturer, Dept. of Mechanical and Manufacturing Engineering, Trinity College, Dublin 2, Ireland. AIAA Member.

${ }^{\dagger} \mathrm{PhD}$ Student, Dept. of Mechanical and Manufacturing Engineering, Trinity College, Dublin 2, Ireland.

${ }_{\ddagger}^{\ddagger}$ Research Assistant, Mller-Breslau-Str. 8, 10623 Berlin, Germany.

$\S$ Research Fellow, Dept. of Mechanical and Manufacturing Engineering, Trinity College Dublin, Dublin 2, Ireland. 
Chung's technique to develop a partial-coherence based technique, that uses five microphones, to extract ejector internal mixing noise from farfield signatures which were assumed to contain the ejector mixing noise, the externally generated mixing noise, and also another correlated mixing noise presumably from the ejector inlet. Wherever there is more than one source, all of these approaches necessitate the location of at least one sensor near one of the sources, e.g. the core noise source, in order to measure that source in isolation. Where there is only one source in the presence of extraneous noise, it has been shown when using Chung's technique, that no direct measure of the source is necessary. Minami and Ahuja ${ }^{10}$ discuss a technique where only farfield measurements are needed to separate any number of correlated sources from extraneous noise, which due to its distributed nature, could be jet noise for example. Previously published work in this area from the 1970's and 1980's has been revisited in more recent years by Hsu and Ahuja ${ }^{9}$ and Nance. ${ }^{11}$ In Bennett and Fitzpatrick, ${ }^{12}$ techniques which can be used to identify the contribution of combustion noise to near and far-field acoustic measurements of aero-engines have been evaluated.

The coherence based source analysis techniques above can be used to identify the contribution of combustion noise in the exhaust of a jet engine and hence enable the design of noise reduction devices. However, when the combustion noise propagates in a non-linear fashion the identified contribution using ordinary coherence methods will be inaccurate. In the paper by Bennett and Fitzpatrick, ${ }^{13}$ an analysis technique to enable the contribution of linear and non-linear mechanisms to the propagated sound to be identified was reported. The technique was then applied to data from a small scale rig and to data from full scale turbo-fan engine tests. In this paper the techniques developed by Bennett and Fitzpatrick ${ }^{13}$ using tonal interactions are extended to examine bandlimited noise. In addition, the quadratic interaction model assumed in the previous paper is enhanced. The techniques are illustrated using simulations before being validated on experimental data from a small bench-test rig. The techniques are subsequently applied to data generated from within an FP7 Collaborative European Aeroacoustics Project: TEENI.

\section{Non-linear Analysis}

Acoustic interaction between rotors is a common observation in turbomachinery noise measurements and has been discussed analytically by Cumpsty,${ }^{14}$ Holste \& Neise ${ }^{15}$ Enghardt et al. ${ }^{16}$ and numerically by Nallasamy. ${ }^{17}$ Energy at two different frequencies may interact to induce energy at a third. In these situations, the upstream energy source is a rotor-stator pair whose excited spinning modes impinge upon a second rotor found downstream. These interactions manifest themselves as sum and difference frequencies. However, the case where noise, which originates from an alternative noise source to the upstream rotor/stator pair, interacts with a downstream rotor-stator pair, is a relatively unexplored area. In Bennett \& Fitzpatrick ${ }^{13}$ it was shown, using an experimental rig, how it is also possible for a tone generated, not from an additional upstream rotor/stator pair, but instead from an upstream speaker, to interact with a downstream rotor/stator

pair to produce interaction frequencies. In addition, analysis of data acquired from full scale aero-engine tests within a European test campaign (Silencer) demonstrated that noise generated from the combustor interacted with both the high pressure and low pressure turbines to generate noise at sum and difference frequencies which were measured in the hot jet pipe of the engine. In this paper, the case where broadband or narrowband noise, such as may originate from a combustor or within the turbomachinery itself, interacts with a rotor-stator pair, to produce noise at sum and difference frequencies, is examined.

\section{II.A. Nonlinear Simulations}

The non-linear analysis of this paper investigates how to accommodate, in addition to propagated combustion noise and turbine noise measured at the exit plane of an aero-engine, the possibility of an interaction between the upstream combustion noise and the turbine, as outlined in figure 1. What is suggested is that the additional inputs into the system due to non-linearities could be an alternative cause for the drop in coherence with increasing engine speed and not simply the relative decrease in importance of the combustion noise compared to the other linear terms.

In order to investigate the influence of the non-linear interactions, a series of simulations were performed using synthetic data. A common non-linear interaction is quadratic in nature resulting in sum and difference frequencies as well a doubling in frequency. This can be demonstrated by observing the following two trigonometric identities. 

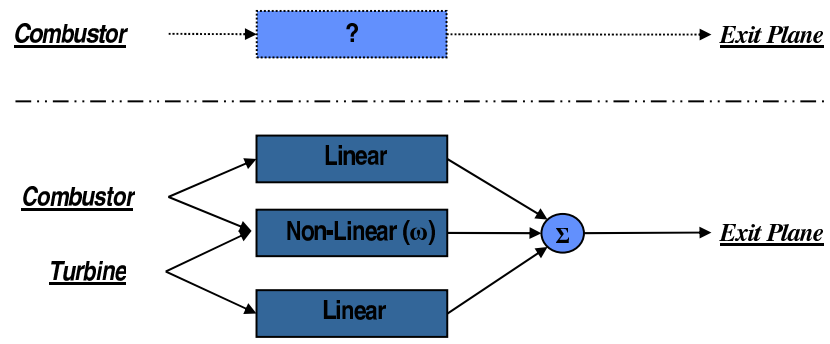

Figure 1. Frequency response function between the combustion noise and the pressure measured at the exit plane when some rpm dependent non-linearity is included in the model.

$$
\begin{aligned}
{[A \cos (\omega t)]^{2} } & =\frac{1}{2} A^{2}[1+\cos (2 \omega t)] \\
{\left[A \cos \left(\omega_{1} t\right)+B \cos \left(\omega_{2} t\right)\right]^{2} } & =\frac{1}{2} A^{2}\left[1+\cos \left(2 \omega_{1} t\right)\right]+\frac{1}{2} B^{2}\left[1+\cos \left(2 \omega_{2} t\right)\right]+A B \cos \left(\omega_{1}+\omega_{2}\right) t+A B \cos \left(\omega_{1}-\omega_{2}\right) t
\end{aligned}
$$

The doubling of frequency arises from self interaction, whereas the sum and difference frequencies come about from combination interactions.

For the basic simulation discussed here, and illustrated in figure 2, synthetic data was generated in Matlab using tones to represent the signals, as they more readily demonstrate the phenomena. To be seen in figure $2(\mathrm{~A})$ is an auto-spectrum of a single tone at $1500 \mathrm{~Hz}\left(G_{c o m b}\right)$, assumed to originate from the combustor can, and a second tone at $2500 \mathrm{~Hz}$, assumed to be generated by the turbine, $\left(G_{\text {turb }}\right)$. The linear superposition of these tones is shown in figure 2 (B). This would be indicative of the auto-spectrum which would be expected to be measured at the exit plane in the absence of nonlinear interaction. Figure $2(\mathrm{C})$ shows the self and combination interactions, $\left(G_{c o m b}+G_{t u r b}\right)^{2}$, which would occur between these linear tones in the presence of a quadratic nonlinearity. Figure 2 (D) shows the exit plane measurement, in black, being the summation of the linear and non-linear contributions when nonlinear interaction is present. Clearly an ordinary coherence measurement between a sensor located near the combustor can measuring only the combustion noise and a sensor located at the exit plane, would be unable to detect the contribution of the combustor noise to the exit plane measurement at the nonlinear interaction frequencies.

\section{II.B. Nonlinear Identification Techniques}

For ideal test situations, an upstream sensor measurement will be able to isolate the local noise source. Figure 3 shows the ideal incident pressure measurements in an aeroengine where a measure of combustion noise, for example, is attainable upstream of a turbine and where the downstream measurement contains information coherent with both the turbine noise, the combustion noise and also the non-linear interactions when present. In real situations, however, such as the full scale engine tests discussed in Bennett and Fitzpatrick ${ }^{13}$ and in the experimental tests presented in this paper, turbine noise and nonlinear interactions will also propagate upstream. The result of this is that both upstream and downstream measurements will contain information coherent with each of the noise sources listed in figure 3(b).

As such, figure 4 is more representative of our situation, where both the upstream measurement $x(t)$ and the downstream measurement $y(t)$ contain both the linear and nonlinear contributions. The challenge, thus, is to identify the presence of nonlinear interactions, such as those illustrated in figure 2 (D), and furthermore to separate these nonlinear contributions from the total exit plane measurement for the scenario depicted in figure 4.

The technique developed in Bennett and Fitzpatrick ${ }^{13}$ to facilitate this was to define a "non-linear" coherence as

$$
\text { Nonlinear Coherence }=\gamma_{\left(x^{2}, y\right)}^{2}
$$

which is simply the ordinary coherence between the square of the upstream measurement with the downstream measurement. The rational behind this is illustrated in an expansion of the square of the upstream measurement, $x(t)$; 

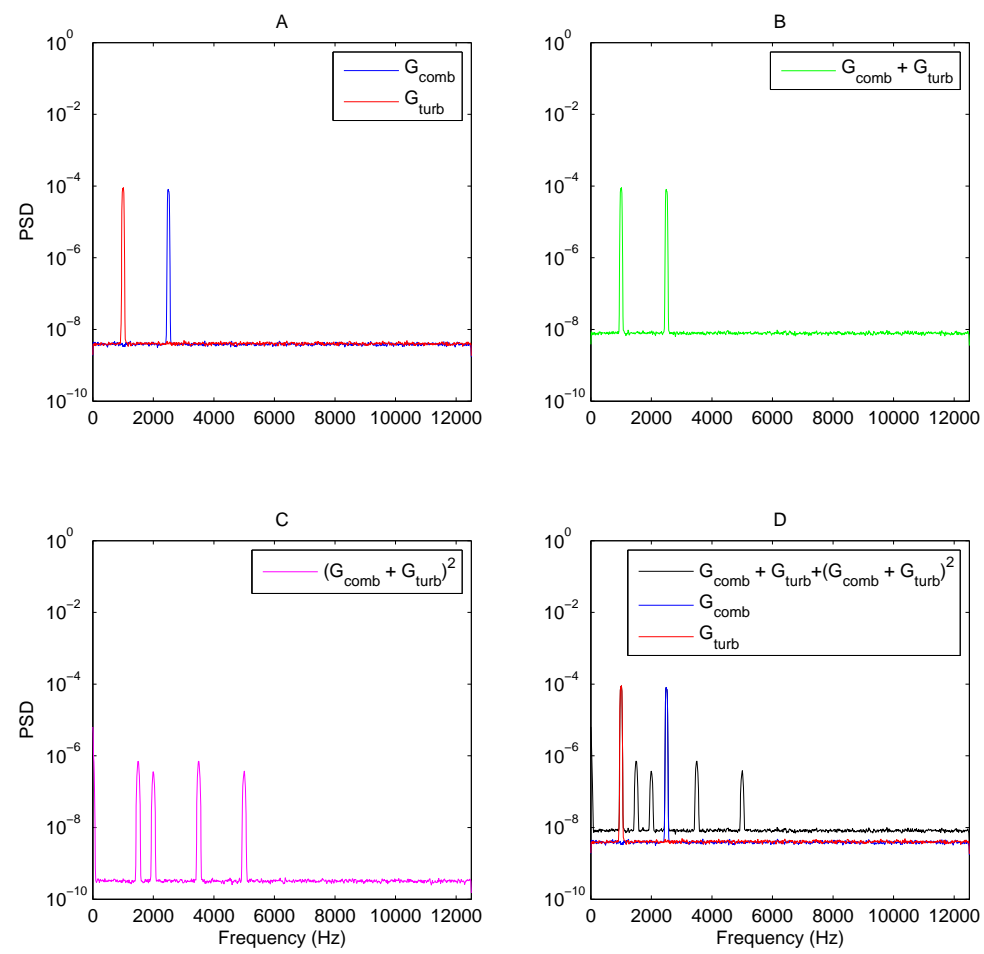

Figure 2. Auto spectra: simulations with single rotor tone, $10 \%$ noise.

\section{$\operatorname{Comb}(t) \longrightarrow H(\omega) \longrightarrow p^{+}{ }_{u p}(t)$}

(a) Incident pressure upstream, $p_{u p}^{+}(t)$

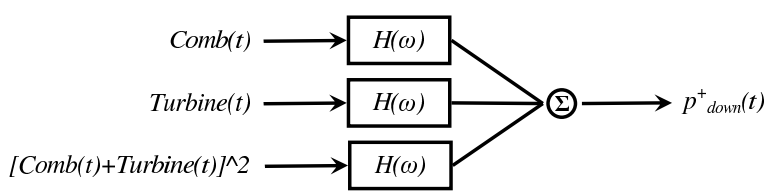

(b) Incident pressure downstream, $p_{\text {down }}^{+}(t)$

Figure 3. Incident pressure models accommodating a quadratic non-linear term.

$$
x=C+T+(C+T)^{2}
$$

given in the following equation ${ }^{\mathrm{a}}$;

$$
\left((C+T)+(C+T)^{2}\right)^{2}=\underline{C^{2}+2 C T+T^{2}}+6 C^{2} T+6 C T^{2}+4 C^{3} T+6 C^{2} T^{2}+4 C T^{3}+2 C^{3}+2 T^{3}+C^{4}+T^{4}
$$

By squaring the upstream measurement, the nonlinear contribution is maintained (underlined) whereas neither of the two linear components, $C$ nor $T$ explicitly remain in the expansion. Therefore, a coherence measure between $x^{2}(t)$ and $y(t)$ will identify the nonlinear components within the downstream measurement.

In order to isolate the linear contribution (in the presence of non-linear interactions), conditional spectral analysis techniques such as those discussed in Bendat and Piersol ${ }^{18}$ and employed by Esmonde et al ${ }^{19}$ may be used. Pertinent to this problem are results where it is calculated that if two arbitrary signals, $i$ and $j$, are composed of at least two components each, where $r$ is one of them, then the cross-spectrum between $i$ and $j$ with the linear effects of $r$ removed can be expressed as

$$
G_{i j \cdot r}=G_{i j}-\frac{G_{i r} G_{r j}}{G_{r r}}
$$

It can be seen from this equation that the part correlated with $r$ must then be

$$
G_{i j: r}=\frac{G_{i r} G_{r j}}{G_{r r}}
$$

a The dependance on $\mathrm{t}$ has been dropped for reasons of clarity 
For $i=j$, the autospectrum of either $i$ or $j$ with the linear part of $r$ removed can be written as

$$
G_{i i \cdot r}=G_{i i}-\frac{G_{i r} G_{r i}}{G_{r r}}
$$

with the correlated part given by

$$
G_{i i: r}=\frac{\left|G_{r i}\right|^{2}}{G_{r r}}
$$

The partial coherence function between $i$ and $j$ with the linear effects of $r$ removed, as derived in Bendat and Piersol, ${ }^{18}$ may now be defined as the ordinary coherence function between the conditioned spectra;

$$
\gamma_{i j \cdot r}^{2}=\frac{\left|G_{i j \cdot r}\right|^{2}}{G_{i i \cdot r} G_{j j \cdot r}}
$$

Applying this to our system, with the "nonlinear" coherence defined as above, a two input/single output model can be constructed as given in figure 5. Using this model, the non-linear contribution can be conditioned from the upstream measurement $x(t)$, to leave the "residual" or partial coherence, viz. the part coherent with the linear components of $y(t)$. This linear coherence for this two input/single output model is given by

$$
\text { Linear Coherence }=\gamma_{(x, y) \cdot x^{2}}^{2}
$$

This technique was used with success in Bennett and Fitzpatrick ${ }^{13}$ as previously discussed. The premise for this technique is that the $x^{2}(t)$ term as expanded in Eqt. (5) is coherent only with the nonlinear contributions of the downstream measurement $y(t)$. Applying this technique to the simulated data of section II.A, we see in figure 6 (A) how the ordinary coherence function fails to separate the linear from the nonlinear terms but how Eqt. (3) applied to this data, in figure 6 (B), amplifies the relative importance of the nonlinear terms. The ideal objective of this technique is to be able to obtain nonlinear and linear coherences such as the theoretical coherences given in figures $6(\mathrm{C})$ and $6(\mathrm{D})$. Whilst the non-linear coherence presented in 6 (B) has suppressed the influence of the linear contributions, it is clear that for this simulation set-up, their contribution is still not insignificant. In this paper the technique is enhanced to improve the definition of the non-linear coherence.

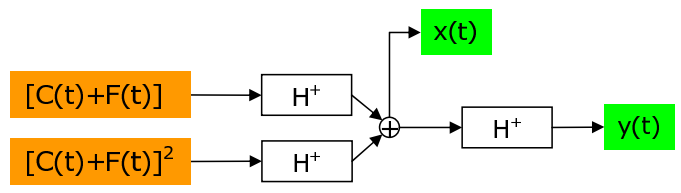

Figure 4. Inputs into upstream and downstream measurements can be modelled as having linear and non-linear parts.

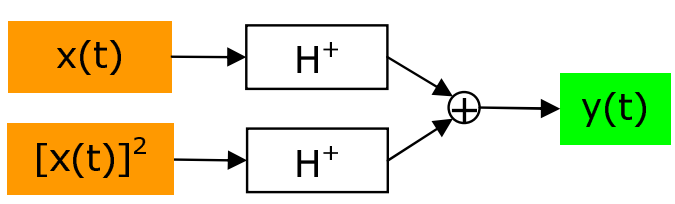

Figure 5. Input/output model for non-linear decomposition

A further examination of the expansion of Eqt. (5), shows that whilst the linear terms $C$ and $T$ do not appear explicitly, certain terms in the expansion are partially correlated with them. The first column of Table 1 highlights, in grey, these terms. An enhanced formulation of the nonlinear coherence would be, therefore, to condition these terms from $x^{2}(t)$. From analysis of the $x^{3}(t)$ expansion, as presented in the second column of Table 1, it can be seen that multiples of the same correlated terms appear. Therefore, to condition $x^{3}(t)$ from $x^{2}(t)$ is to remove these partially coherent terms. However, the expansion of $x^{3}(t)$ also contains further terms not contained in the $x^{2}(t)$ expansion, and therefore, additional useful information would also be removed. A more optimal approach can be found by conditioning using the $x^{4}(t)$, (column 3 of Table 1) from $x^{3}(t)$ term first and then to condition this residual from $x^{2}(t)$. Conditional Spectral Analysis is well suited to such treatment of inputs and figure 7 (A) presents the new multiple input/single output model for the nonlinear coherence. To remove this new nonlinear residual from the $x(t)$ term input is therefore to obtain the linear coherence. The model to obtain the linear coherence is given in figure 7 (B).

Results using these new formulations of the nonlinear and linear coherences are shown in figure 8. By conditioning the $x^{2}(t)$ term with only the $x^{3}(t)$ we see in figure $8(\mathrm{~A})$ that the contributions of the linear terms have been reduced, and indeed this is an improvement of the result using just the $x^{2}(t)$ term as per 
figure $6(\mathrm{~B})$. However, figure $8(\mathrm{C})$ shows how the influence of the linear terms can be entirely removed by first conditioning using the $x^{4}(t)$ term. Notice that the value of the coherence at the frequencies of the non-linear terms reduces with each conditioning. In parallel, the linear coherence for each permutation is shown in figures 8 (B) and (D) and is seen to be close to the ideal situation in both cases. Given these new advances, the nonlinear and linear coherences are redefined as

$$
\begin{aligned}
\text { Enhanced Nonlinear Coherence } & =\gamma_{\left(x^{2}, y\right) \cdot x^{3} \cdot x^{4}}^{2} \\
\text { Enhanced Linear Coherence } & =\gamma_{(x, y) \cdot x^{2} \cdot x^{3} \cdot x^{4}}^{2}
\end{aligned}
$$

and are shown schematically in figure 7.
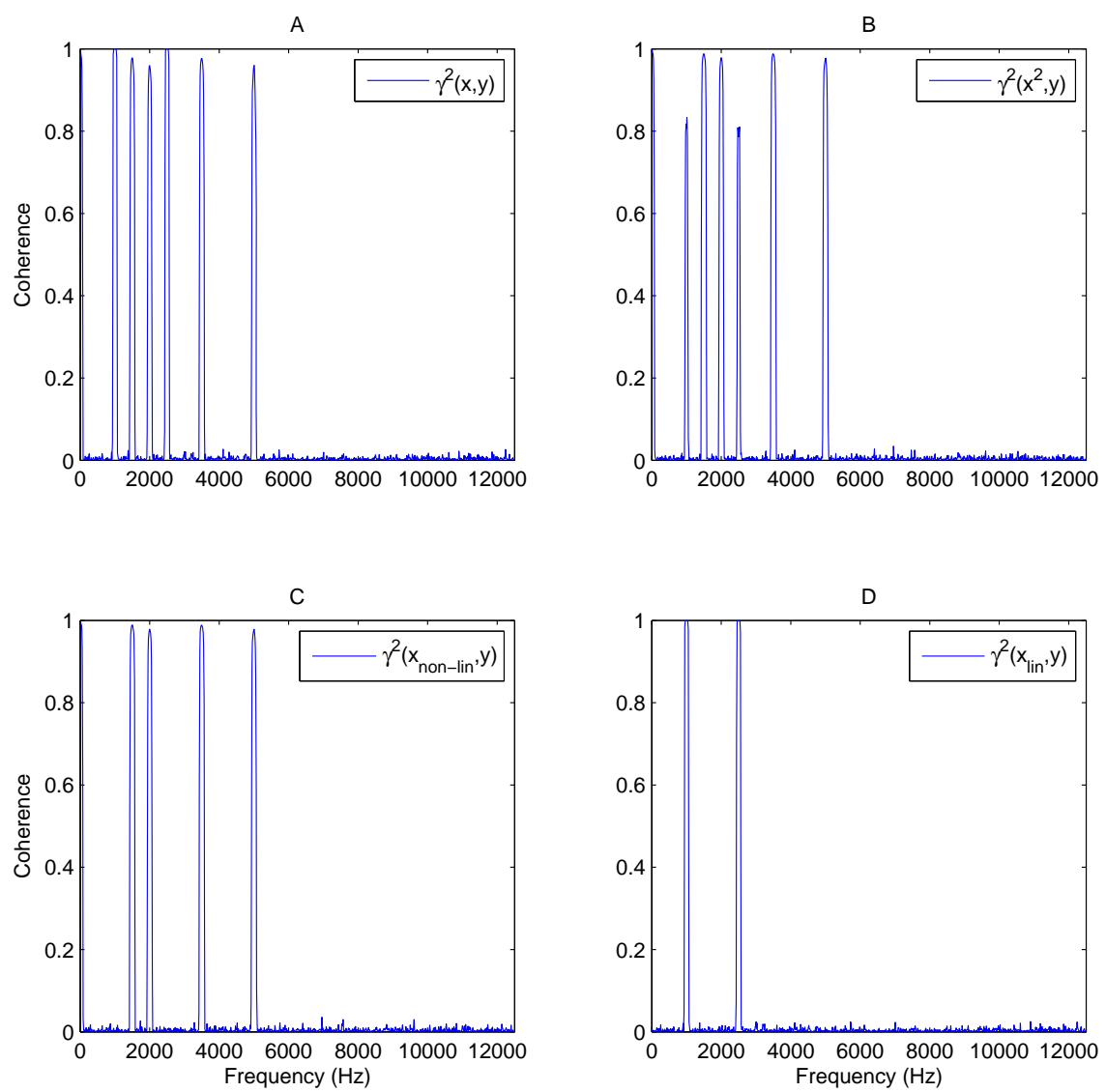

Figure 6. Ordinary coherence (A) and non-linear coherence (B) applied to the simulated data. Figures 6 (C) and (D) show the theoretical non-linear and linear coherences for the data. $10 \%$ Noise.

\section{Experimental Results}

\section{III.A. Experimental Setup: Bench-Test}

A small laboratory-based experimental rig was designed and built to gain a fundamental physical understanding of the convection of noise through a rotor/stator set up, and is shown schematically in Fig. 9 . A speaker represents the source noise which is directed down a brass tube of $3 \mathrm{~mm}$ wall thickness with an internal diameter of $0.051 \mathrm{~m}$. This end of the tube is open and allows air to be sucked down the pipe by a vane-axial fan situated at a minimum of $1.2 \mathrm{~m}$ from the entrance, according to the test set-up. This vane-axial fan which has a single 8 blade rotor stage downstream of a single 5 vane stator stage represents a simplified turbine of a turbofan engine. The tube ends with an open anechoic termination designed to reduce flow expansion/separation noise as well as reflections. A number of microphones were located upstream and downstream of the fan, at various axial positions, such that they were mounted flush with the inside of the pipe. 


\begin{tabular}{|c|c|c|}
\hline$x^{2}$ & $x^{3}$ & $x^{4}$ \\
\hline$C^{2}$ & $20 C^{3} T^{3}$ & $120 C^{3} T^{3}$ \\
$2 C T$ & $6 C^{5} T$ & $36 C^{5} T$ \\
$T^{2}$ & $6 C T^{5}$ & $36 C T^{5}$ \\
$6 C^{2} T$ & $3 C^{2} T$ & $T^{8}$ \\
$6 C T^{2}$ & $3 C T^{2}$ & $C^{8}$ \\
$6 C^{2} T^{2}$ & $18 C^{2} T^{2}$ & $6 C^{2} T^{2}$ \\
$4 C^{3} T$ & $12 C T^{3}$ & $4 C T^{3}$ \\
$4 C T^{3}$ & $12 C^{3} T$ & $4 C^{3} T$ \\
$2 C^{3}$ & $C^{3}$ & $8 C T^{7}$ \\
$2 T^{3}$ & $T^{3}$ & $8 C^{7} T$ \\
$C^{4}$ & $15 C^{4} T^{2}$ & $90 C^{4} T^{2}$ \\
$T^{4}$ & $15 C^{2} T^{4}$ & $90 C^{2} T^{4}$ \\
- & $3 C^{5}$ & $4 C^{5}$ \\
- & $3 T^{5}$ & $4 T^{5}$ \\
- & $3 C^{4}$ & $C^{4}$ \\
- & $3 T^{4}$ & $T^{4}$ \\
- & $15 C T^{4}$ & $20 C T^{4}$ \\
- & $15 C^{4} T$ & $20 C^{4} T$ \\
- & $C^{6}$ & $6 C^{6}$ \\
- & $T^{6}$ & $6 T^{6}$ \\
- & $30 C^{3} T^{2}$ & $40 C^{3} T^{2}$ \\
- & $30 C^{2} T^{3}$ & $40 C^{2} T^{3}$ \\
- & - & $56 C^{3} T^{5}$ \\
- & - & $56 C^{5} T^{3}$ \\
- & - & $28 C^{6} T$ \\
- & - & $28 C T^{6}$ \\
- & - & $70 C^{4} T^{4}$ \\
- & - & $84 C^{5} T^{2}$ \\
- & - & $140 C^{4} T^{3}$ \\
- & - & $140 C^{3} T^{4}$ \\
- & - & $28 C^{6} T^{2}$ \\
- & $28 C^{2} T^{6}$ \\
- & $4 C^{7}$ \\
- & - & $4 T^{7}$ \\
\hline
\end{tabular}

Table 1. $\mathrm{x}(\mathrm{t})$ term expansions

The data was acquired using a National Instruments 48 channel, 24 bit data acquisition system. Each channel was simultaneously sampled and automatically low pass filtered to avoid aliasing. The acquired data was stored on a personal computer prior to post processing.

\section{III.A.1. Narrowband Interaction}

An analysis was performed on the experimental rig to detect the presence of non-linearities. In order to investigate the hypothesis that upstream broadband noise might interact with a rotor/stator pair to produce acoustic energy at sum and difference frequencies, band limited noise was radiated from the speaker. Figure 10 shows the sound pressure level at a location downstream of the vane-axial fan using a microphone flush 

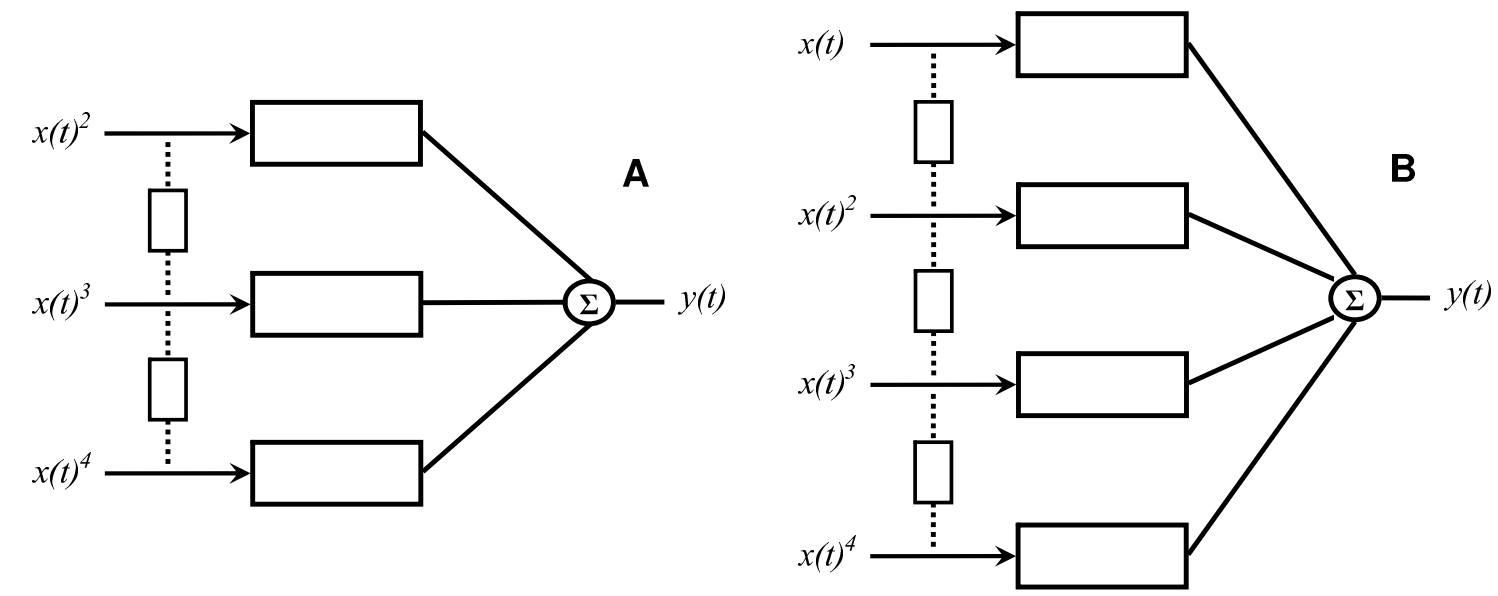

Figure 7. Conditional Spectral Analysis technique schematics for nonlinear and linear partial coherence models.
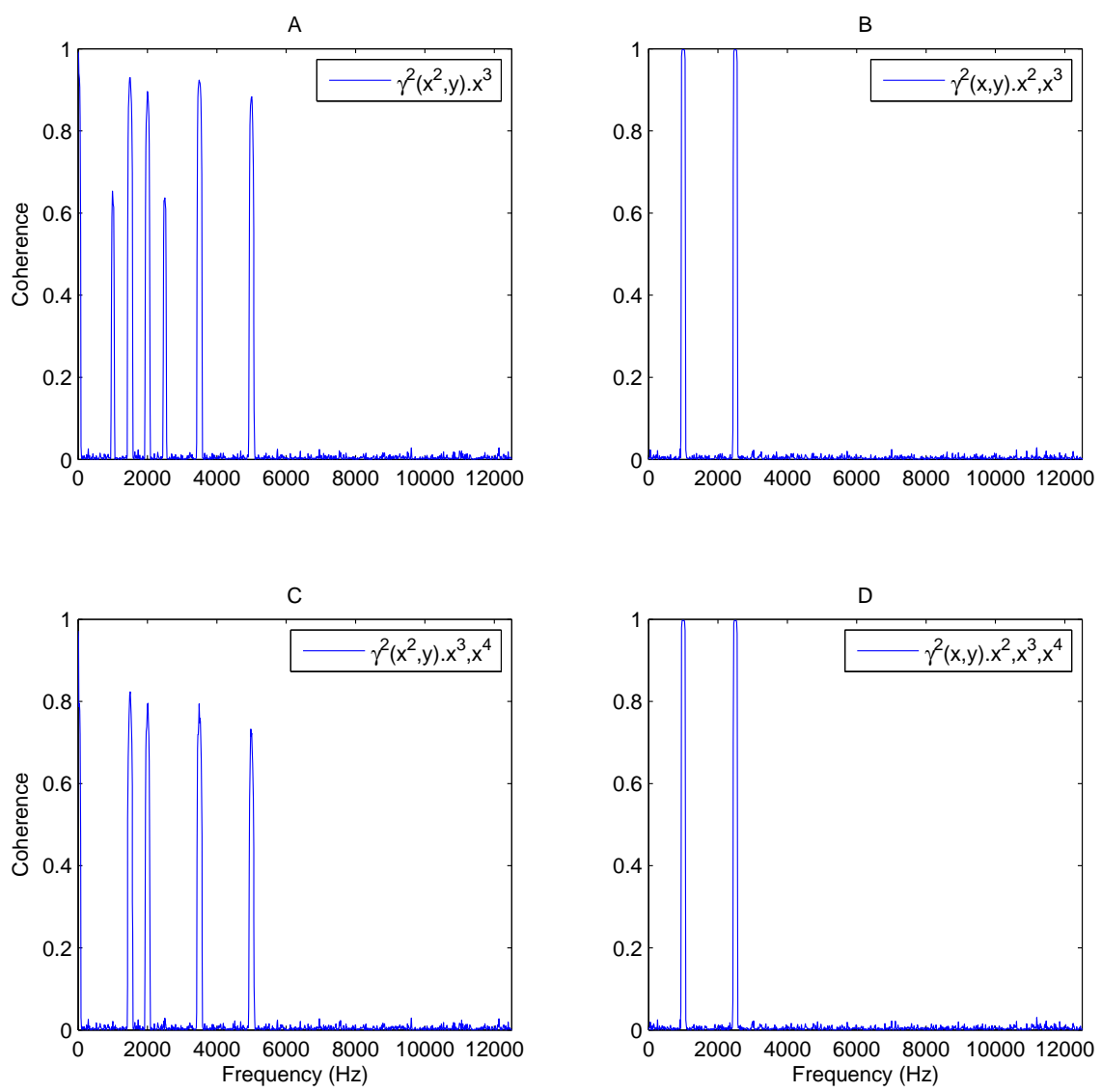

Figure 8. Enhanced nonlinear and linear coherences applied to simulated data. $10 \%$ noise.

mounted with the inside of the duct. Three test configurations are shown. The first power spectral density (PSD) in red shows the energy measured by the microphone when band limited noise of $10.5-11 k \mathrm{~Hz}$ is emitted from the speaker. The plot in green shows the PSD when the speaker is turned off and the vane-axial fan is turned on. The fan has a high rotational speed of 16500rpm at the nominal max design voltage of 26VDC. As the fan has 8 blades this would result in a nominal blade pass frequency (BPF) of $2200 \mathrm{~Hz}$. In this test the rpm is lower with the BPF occuring at approximately $1600 \mathrm{~Hz}$ a peak at which can be seen in the figure, as are its harmonics, nBPF, four of which are visible and are indicated with black dashed lines. The third test configuration in this figure is for the case where both the vane-axial fan and speaker operate 


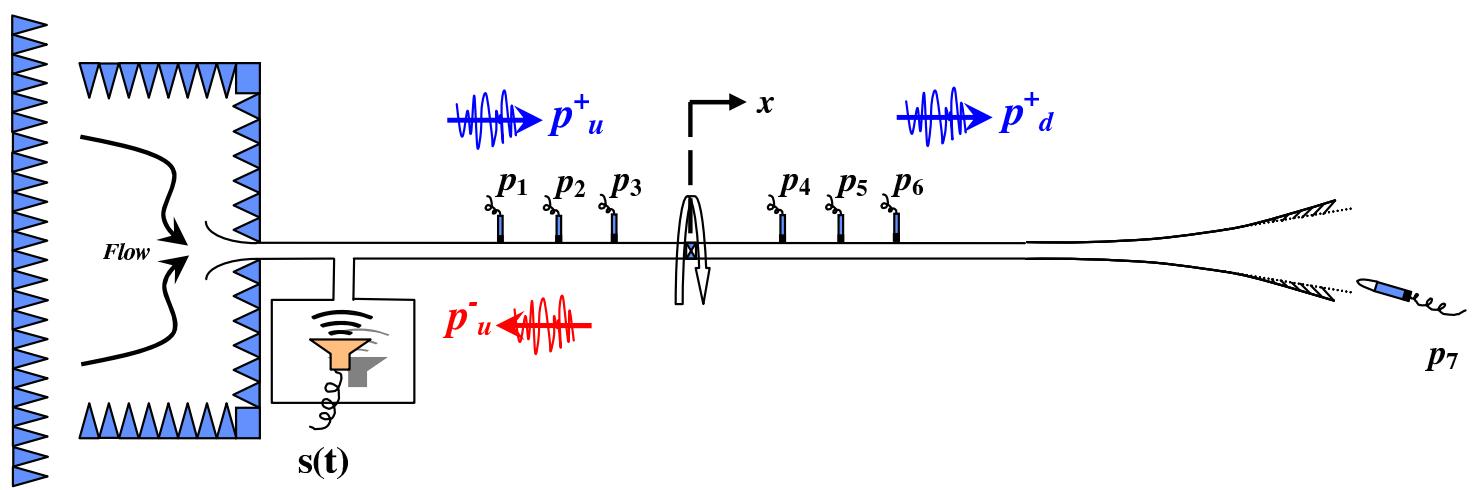

Figure 9. Schematic of experimental set up .

simultaneously. It is clear, from observation of the blue PSD, that in addition to the $500 \mathrm{~Hz}$ band of energy emitted from the speaker, bands of noise are frequency scattered to either side in accordance with $\pm B P F$. This result is highly significant as noise is generated in frequency ranges which cannot be attributed to the simple superposition of the noise sources.

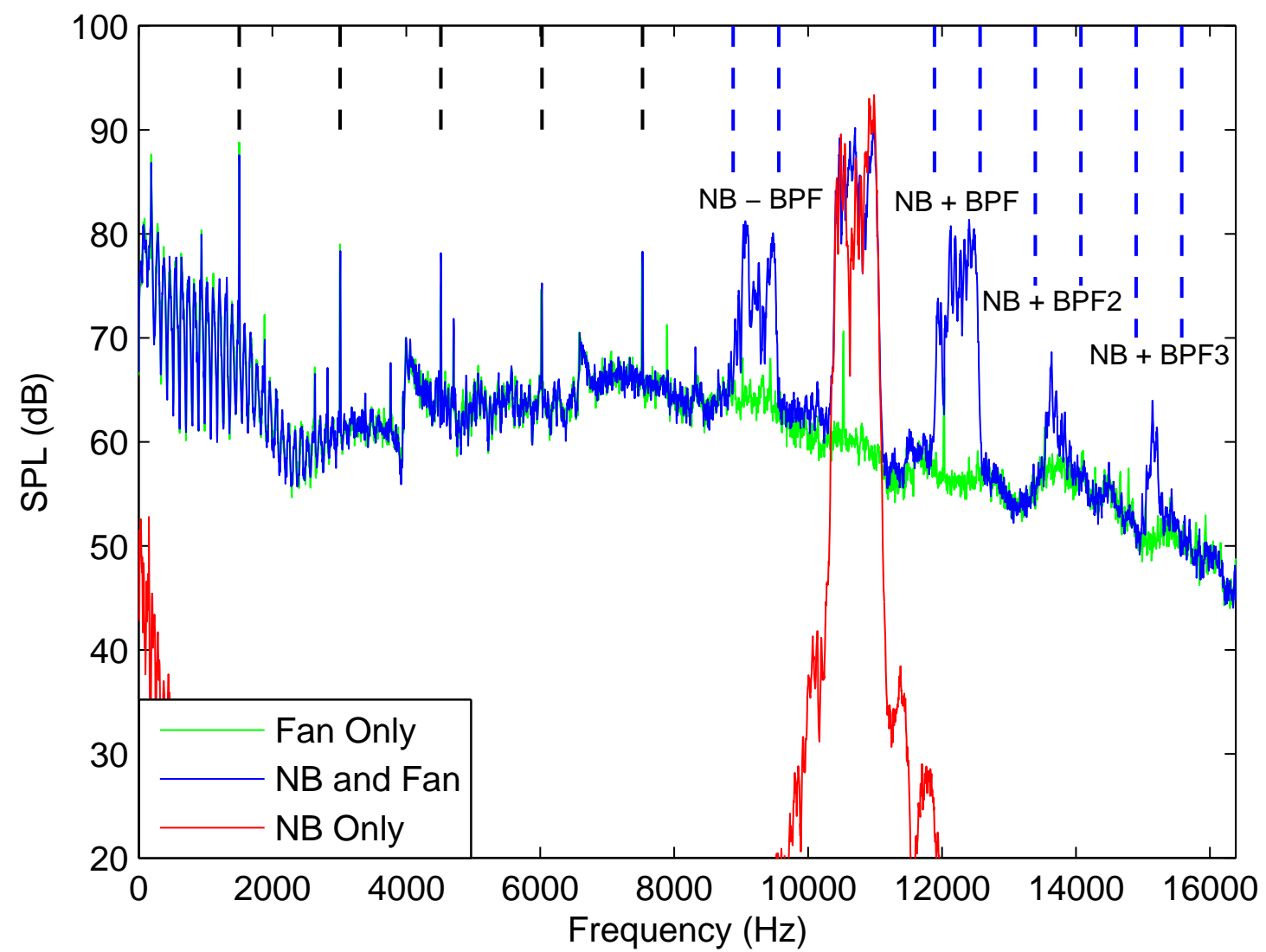

Figure 10. Auto-spectrum showing non-linear interaction between bandlimited noise at $10.5-11 \mathrm{kHz}$ and the fan.

To demonstrate the effect of fan speed and associated $n B P F$ dependency of the frequency scattering, a series of tests were carried out where the narrowband noise source remained constant with a reducing fan speed. Figure 11 shows a waterfall plot of PSD's for the same microphone location but where in each successive test the fan speed is decreased. Two principle observations may be made from this plot. The first is that with decreasing $n B P F$ the sum and difference frequency bands group closer to the speaker source band as to be expected. The second is that as a side effect of decreasing fan speed, the background flow 
noise decreases and additional scattered frequency ranges are revealed.

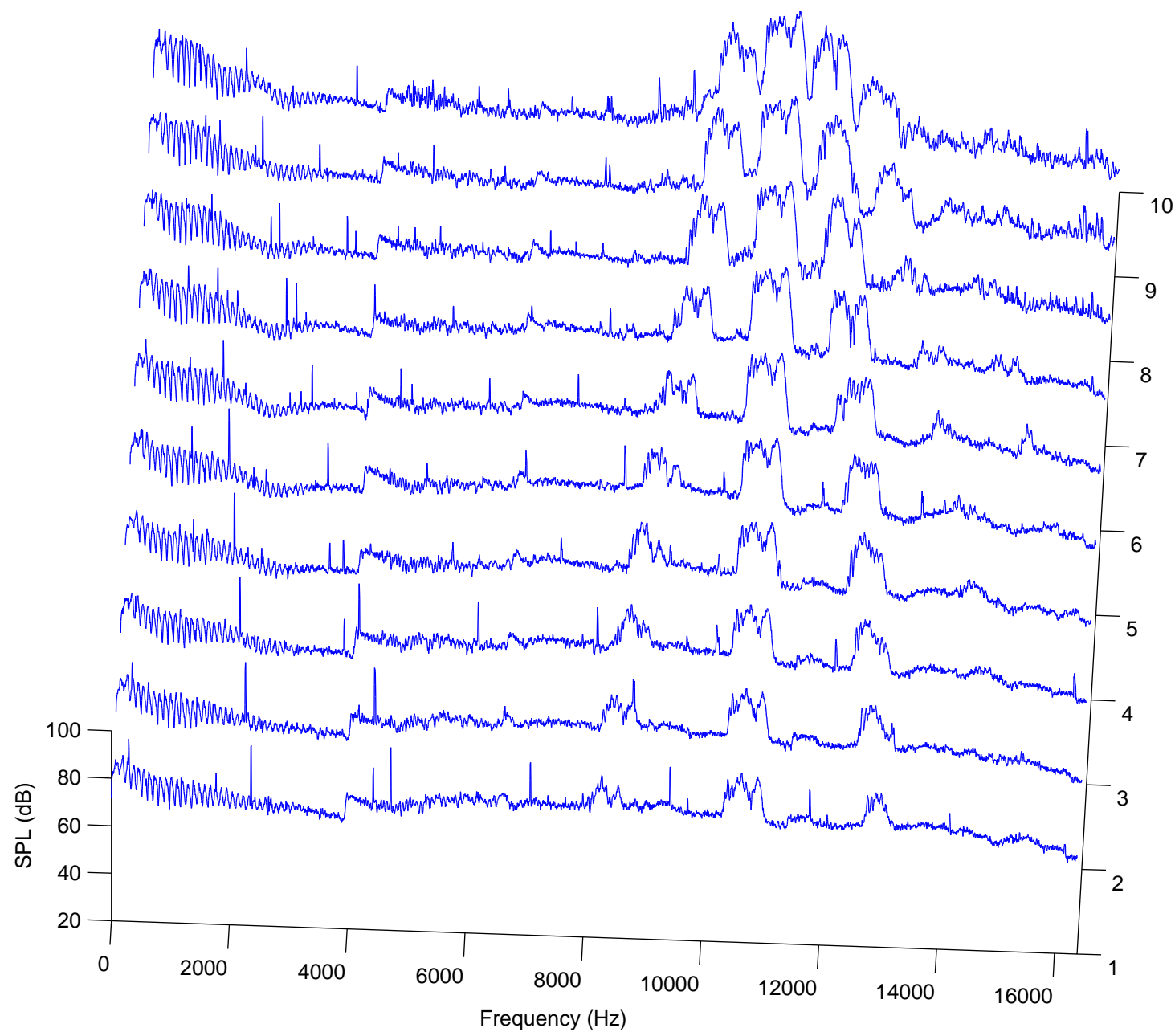

Figure 11. Waterfall plot of auto-spectrum showing non-linear interaction between bandlimited noise and the fan for a number of fan speeds.

This latter point is further examined in figure 12 where the lower noise floor is more clearly seen. Of particular interest in this plot is how the interaction frequency magnitudes can remain significantly high even when the BPF magnitude has decreased substantially. The consequence of this is that nonlinear interaction noise can be a significant contributor to the overall sound pressure and may even be greater than the "linear" noise sources.

\section{III.A.2. Non-linear Identification}

The nonlinear identification techniques of section II are applied here to the experimental data. As discussed, often in real experimental tests, data acquired from sensors contain information from a number of different sources. In such cases, alternatives to the ordinary coherence function need to be applied. In figure 13 (A) and (B), a test set-up is such that microphones located both upstream and downstream of the vane-axial fan contain the vane-axial fan (or "turbine") noise, the speaker (or "combustor") noise and the nonlinear interactions. From observation of figure $13(\mathrm{C})$, the ordinary coherence function between the upstream and downstream microphones reveals little other than that the coherence is high between the two locations for each of the sources. However, if the enhanced nonlinear coherence of Eqt. (12) is employed, then the coherence indicates both that nonlinear interaction has taken place and also identifies the associated frequency ranges.

The usefulness of this techniques as an identification tool is demonstrated in figure 14, where a waterfall 


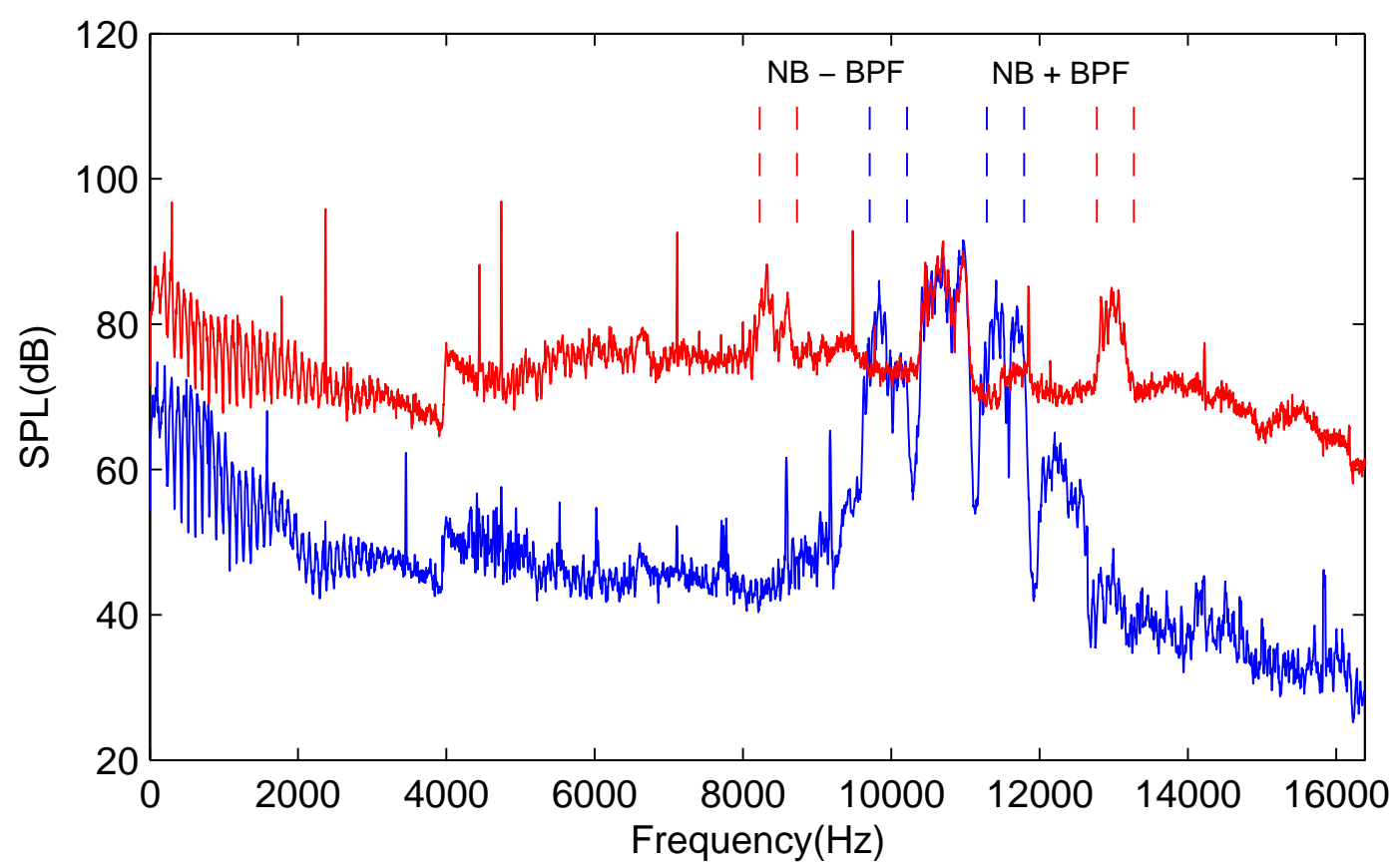

Figure 12. Auto-spectrum showing non-linear interaction between bandlimited noise and the fan at high and low fan speeds.

plot of a new set of data is presented. In this series of tests, the vane-axial fan speed remains constant, and instead, the center frequency at which the bandlimited noise is emitted is incrementally increased. Some evidence of the presence of the nonlinear interactions is evident from the PSD's, however, as previously stated, in full scale turbomachinery tests where there are many rotor/stator stages, such manual diagnosis is unreasonable. The enhanced nonlinear coherence has again been applied to a selection of the PSD's of figure 14, and the results are presented in figure 15. Once again, the ability of the technique to identify the nonlinear interactions, when present, is displayed. The first plot is a situation where there is in fact no non-linear interaction, and for this result the coherence is low at all frequencies.

For all tests carried out on this particular experimental rig, nonlinear interaction has never been observed below a frequency of approximately $7 \mathrm{kHz}$ or when the speaker source frequency is below this critical frequency. In the first plot of figures 14 and 15 , the $500 \mathrm{~Hz}$ bandlimited source with a center frequency of 6250 results in no nonlinear interaction. In the work of Bennett, ${ }^{20}$ the possibility that it is the modal content of the nonlinear interactions that determines its subsequent propagation down the duct was explored. It may also be possible that the diameter length scale, whose wavelength corresponds to the critical $\approx 7 k H z$ cut-off frequency, may be the cause. Both of these hypotheses were further explored within the FP7 European Project TEENI on an experimental rig of DLR, one of the consortium partners. This set-up has a larger diameter duct which would cause the nonlinear interactions to occur at lower frequency ranges such as those in which direct and indirect combustion noise propagates. In addition, as the rig is instrumented with microphone arrays, an acoustic modal decomposition would allow the scattering cut-off frequency hypothesis to be explored.

\section{III.B. Experimental Setup: Small Scale Tests}

The DLR test rig configuration for the TEENI tests is shown in figure 16, and provides adequate test conditions to investigate propagation effects typical for turbo-machinery. A loudspeaker section is used to simulate tonal, narrowband or broadband sound fields of different signatures, representing e.g. direct and indirect combustion noise that is emitted by the combustor/hp turbine section of the turbo-shaft engine. The following parameters can be varied: frequency bandwidth, spectral characteristic, mode content and mode coherences. In order to de-correlate modes of high azimuthal orders, up to 16 loudspeakers placed equidistantly in a single ring around the duct wall can be used. As indicated in the figure, the inner 

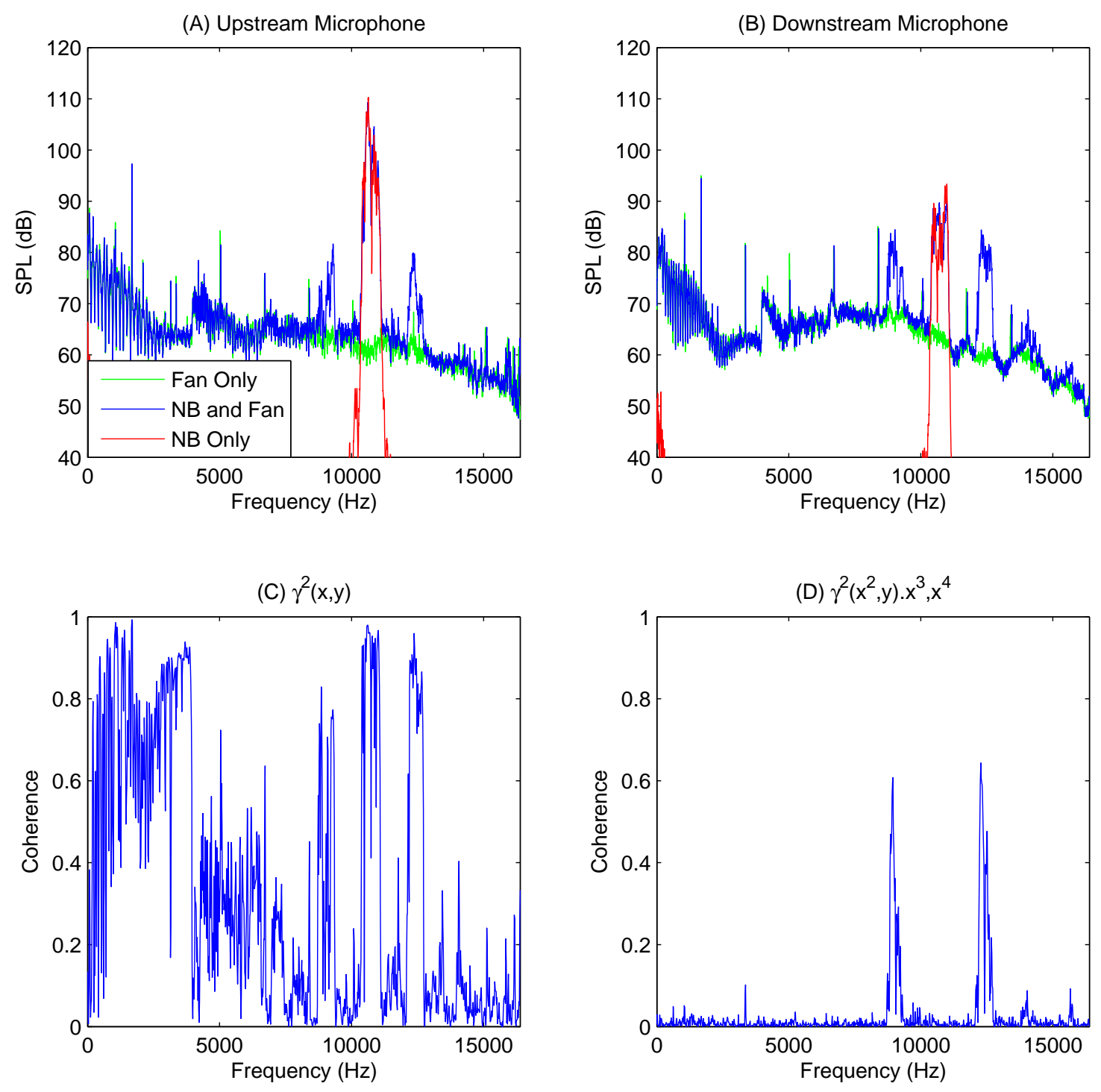

Figure 13. Auto spectra, Non-linear Coherence experimental data

sensor array is used to measure the modal sound field emitted by the simulated combustor noise source and the exhaust sensor array is used to assess the modes that are transmitted through the fan stage. Figure 17 illustrates how the principal features in the rig map onto a typical aeroengine instrumented with a microphone array at the exhaust, as is the intention within the full scale test work package of TEENI. With regard to figure 18, in the given setup the axial fan represents both the mode scattering element as well as an additional source (S1) of both tonal and broadband noise. It should be noted that a hub was introduced upstream (exhaust) of the fan in order to modify the conditions for mode propagation.

The techniques developed in section II.B were applied to data from the DLR rig. Initially, tonal noise was analysed as it is clearer to diagnose and tends to be coherent throughout the duct. A sensor located near the loud speaker section was used to condition data from a microphone located in the exhaust of the duct. This would be equivalent to relating a sensor located in the combustor of an aeroengine to an exit plane measurement. In figure 19, sum frequencies can be seen in both autospectra, indicating that noise from the loudspeaker section has been frequency scattered by the rotor. Focussing on the first sum frequency, the enhanced non-linear coherence technique is seen in figure 20 to successfully separate the linear and nonlinear contributions to the downstream measurement. It can be seen that even though the amplitude of the scattered tone in the vicinity of the loudspeaker section is relatively small, and that the ordinary coherence is, allbeit convincing, equal to 0.5 , the technique clearly identifies the tone as being the result of a non-linear 


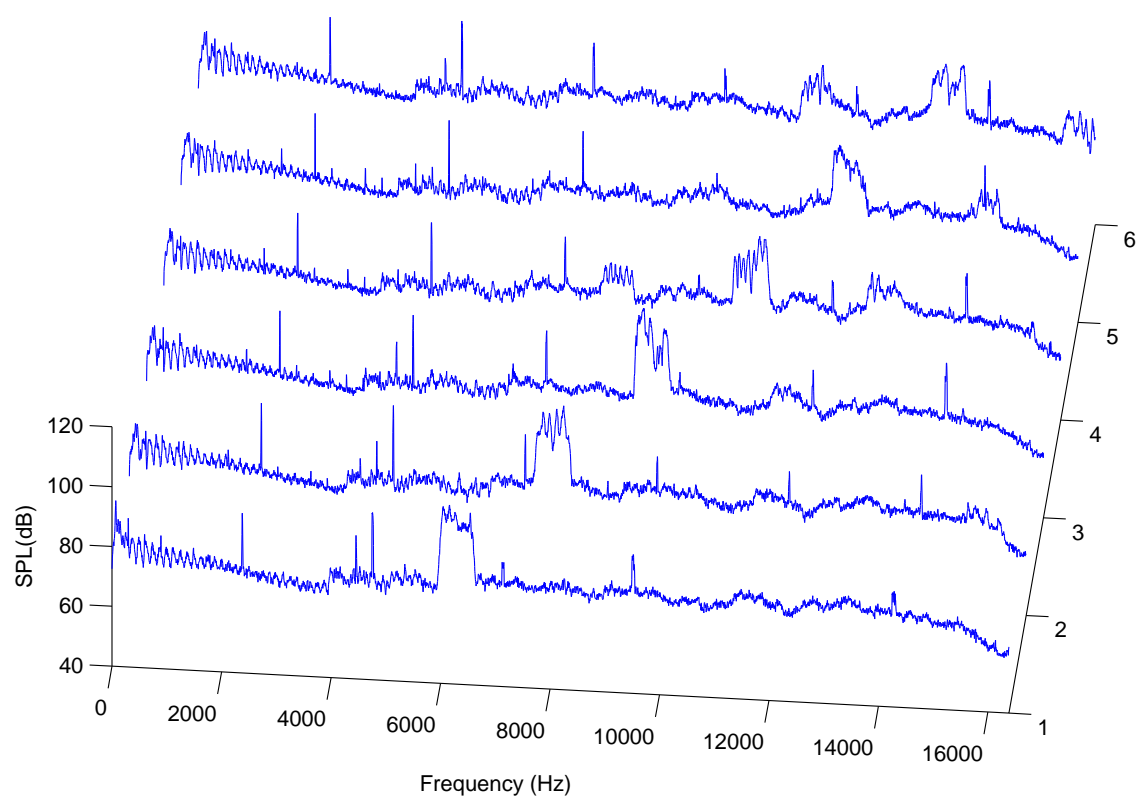

Figure 14. Waterfall plot of downstream microphone auto-spectra showing non-linear interaction between bandlimited noise and the fan for a range of bandlimited frequencies.

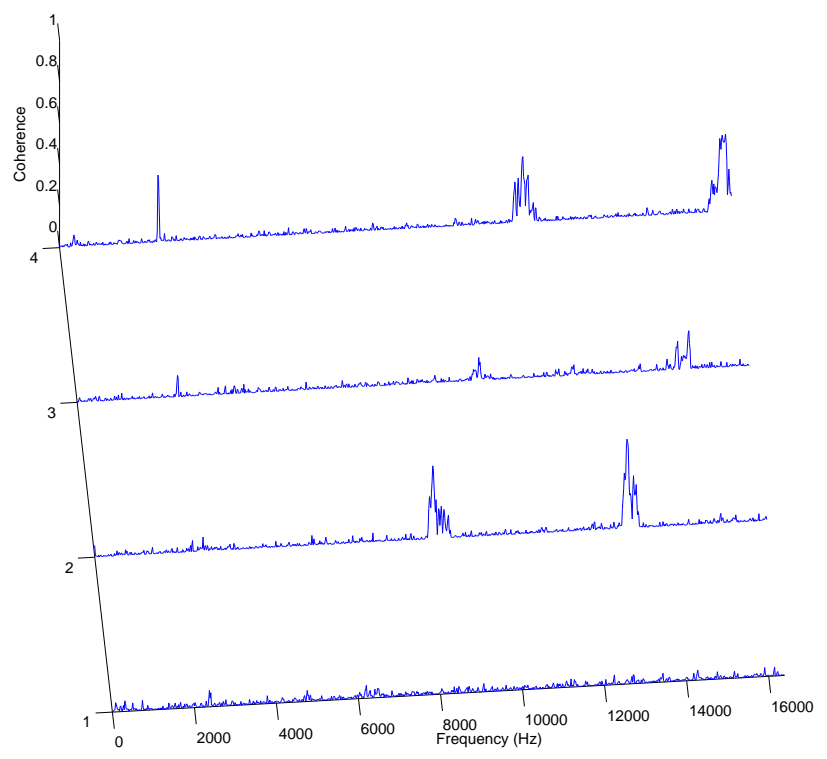

Figure 15. Waterfall plot - Nonlinear coherence applied.

interaction, calculating a non-linear coherence of nearly unity for that frequency and close to zero for the linear contribution.

The technique was then used to address narrowband noise. A similar approach was taken with a different test-point, where narrowband noise centred at $4500 \mathrm{~Hz}$ was emitted from all sixteen loudspeakers in a single ring. As can be seen in figure 21, frequency scattering is again evident for narrowband noise. Very clear regions of energy are frequency scattered into sum and difference frequencies by the blade pass frequency and also by its harmonics. Once again, as seen in figure 22 the non-linear technique is successful in identifying the frequency ranges into which energy is frequency scattered by the non-linear interaction. Broadband/narrowband noise is typically less spatially coherent in a duct and this is evident from looking initially at the ordinary coherence function. Despite the fact that the narrow band energy is dominant at 


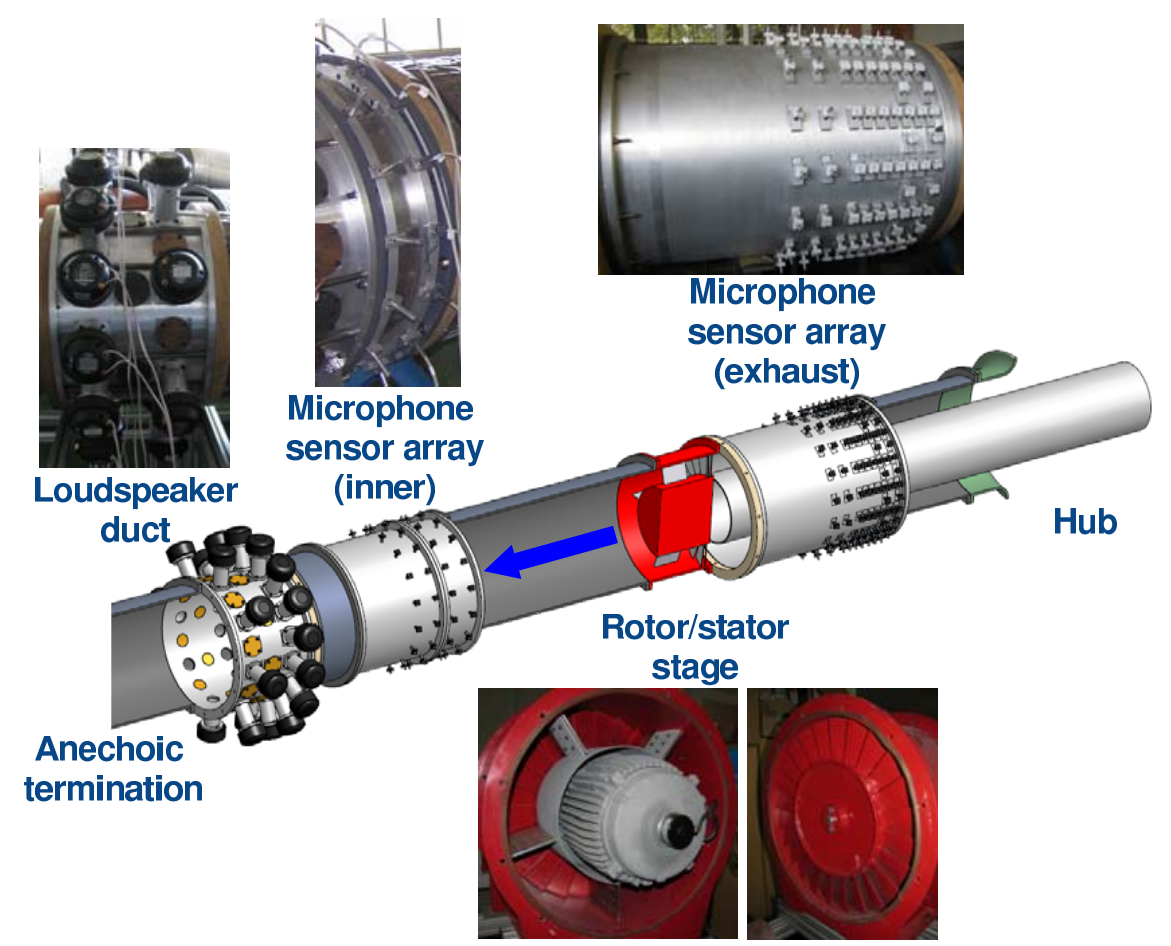

Figure 16. Experimental setup: small scale test. Loudspeaker array and axial fan represent two uncorrelated sources.

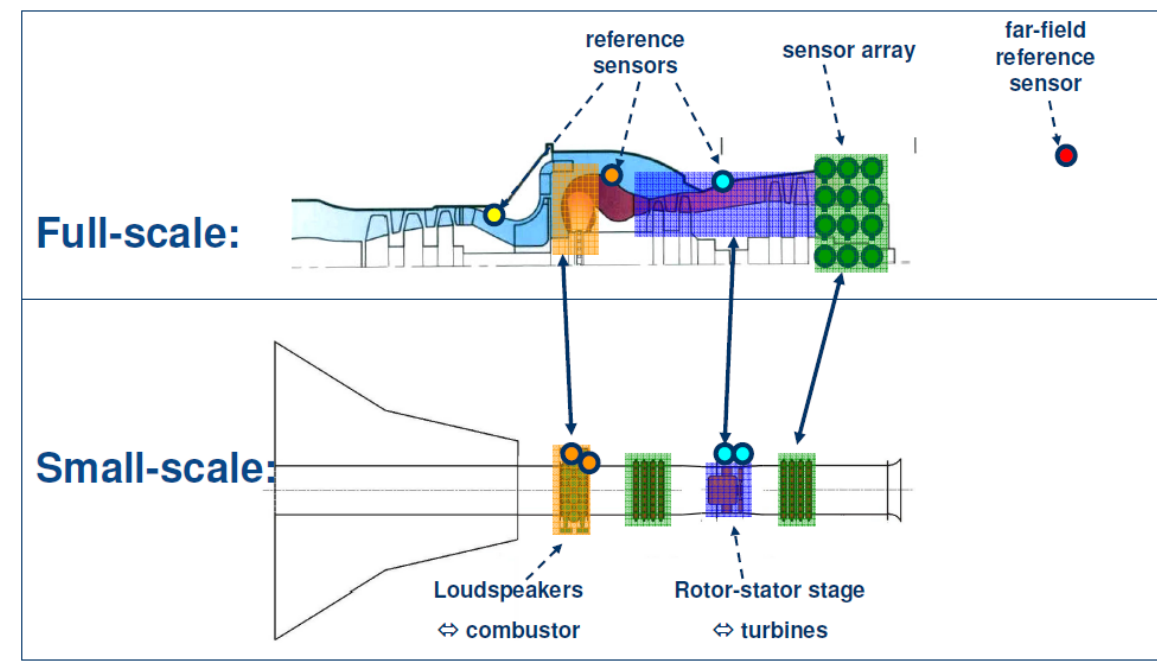

Figure 17. Similarities between the small scale test set-up and a full scale engine.

the two sensor locations, the ordinary coherence at these frequency ranges is not close to unity as might be expected for tonal noise. Despite the low spatial coherence, the non-linear technique performs well, identifying the non-linear components. Low frequency tones are to be seen in the coherence functions but these are attributed to harmonics of the shaft rotational speed and as such are due to sensitivities of the microphones to vibration - which in essence are extraneous noise/non-linear contributions. For the narrowband noise, the identification of the linear component is less convincing. More work needs to be done in this area to fully explain why.

Figure 23 presents a similar waterfall plot to that generated using data from the bench-test rig in figure 14. In this series of tests, the rotor rpm and the bandwidth of the narrowband noise were kept constant, while the centre frequency of the narrowband noise was reduced. At high centre frequencies, frequency scattering is clearly evident and a doubling of the centre frequency can be seen for the low centre frequency test points. However, once again, at these low centre frequency test points there is no obvious measure 


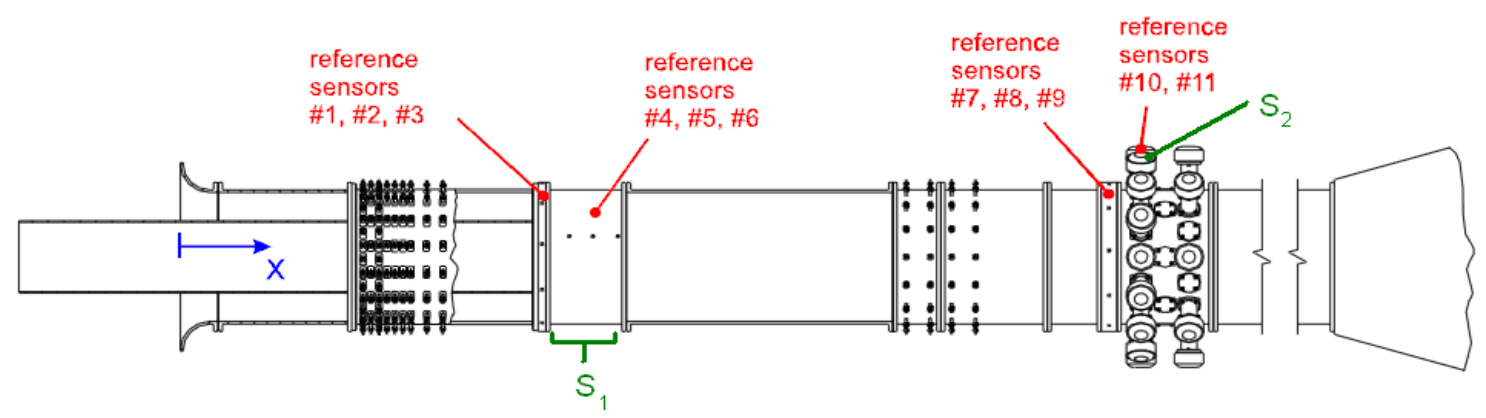

Figure 18. Experimental setup: small scale test. Reference sensor and source locations. S1 = rotor-stator stage. S2 = loudspeaker section generating broadband, narrowband or tonal noise.

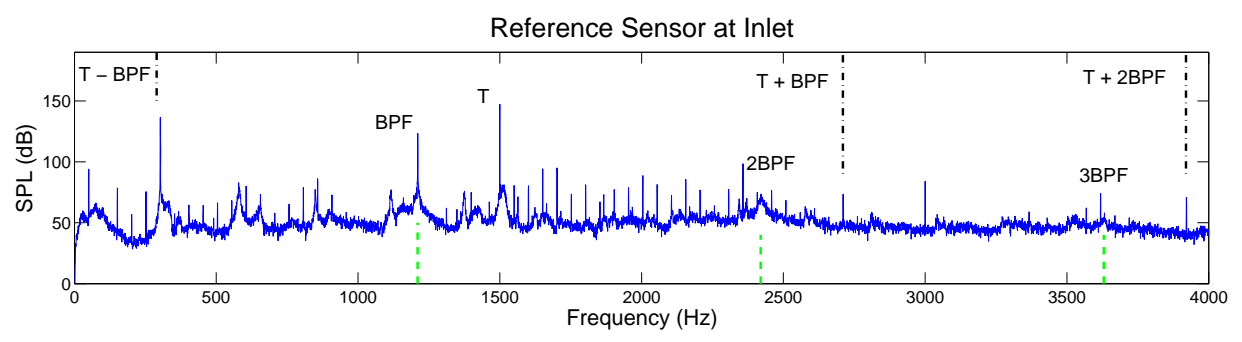

Reference Sensor \#7

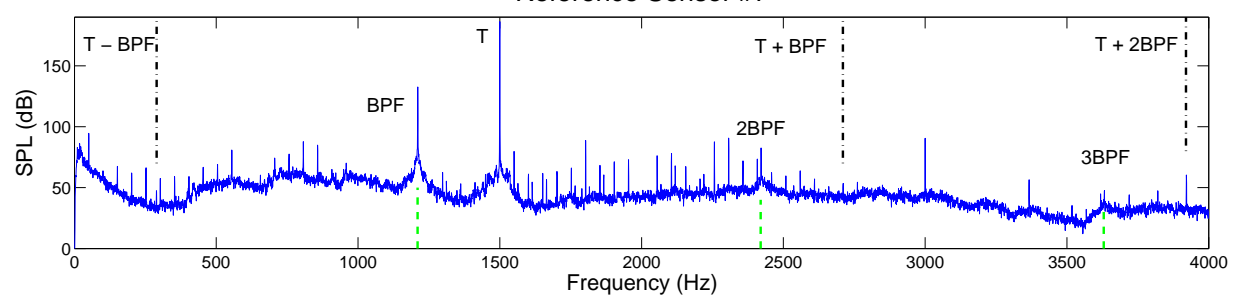

Figure 19. Autospectra of data from two microphones located remotely from each other. One in the exhaust array and the second located near the loudspeaker section. The rotor $\mathrm{rpm}$ is $3000(1200 \mathrm{~Hz})$ and a single tone at $1500 \mathrm{~Hz}$ is emitted from all sixteen loud speakers in a single ring. Sum tones between the BPF and the loudspeaker tone are evident.
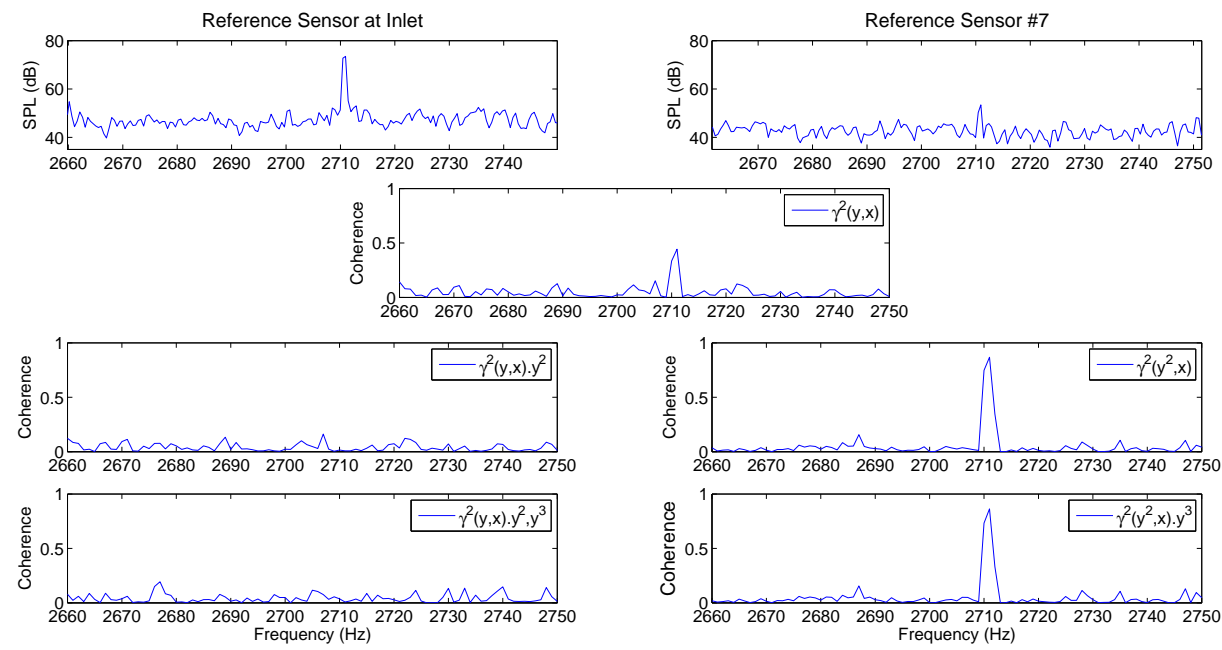

Figure 20. Enhanced non-linear coherence techniques applied to the data of figure 19. Shown is a close-up of the autospectra, the ordinary coherence and also the linear and non-linear components.

of sum and difference interaction. As hypothesized, the larger diameter duct has reduced the "scattering 


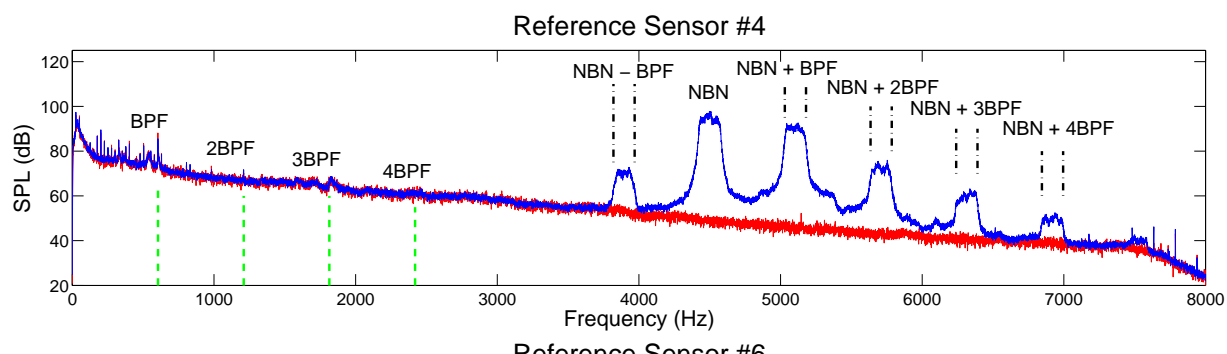

Reference Sensor \#6

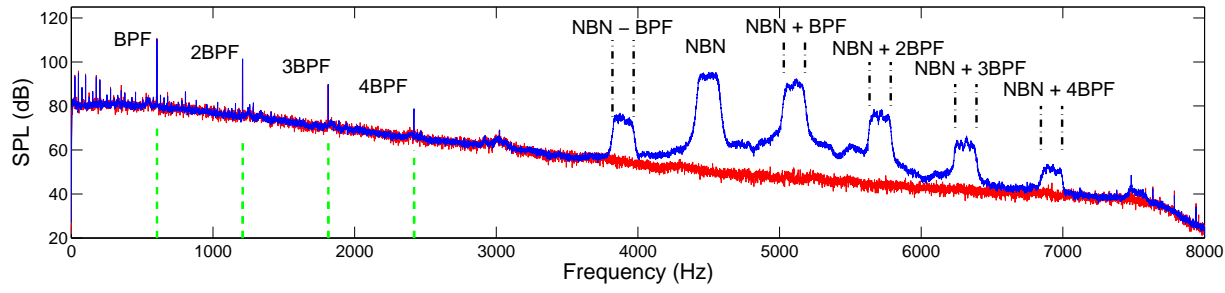

Figure 21. Autospectra of data from two microphones located on either side of the rotor. The rotor $\mathrm{rpm}$ is $1500(600 \mathrm{~Hz})$ and narrowband noise centred at $4500 \mathrm{~Hz}$ with a bandwidth of $150 \mathrm{~Hz}$ is emitted from all sixteen loud speakers in a single ring. The narrowband noise is seen to be frequency scattered into sum and difference frequencies. Autospectra are superimposed onto the plots for the test point where the narrowband noise is turned off (red).
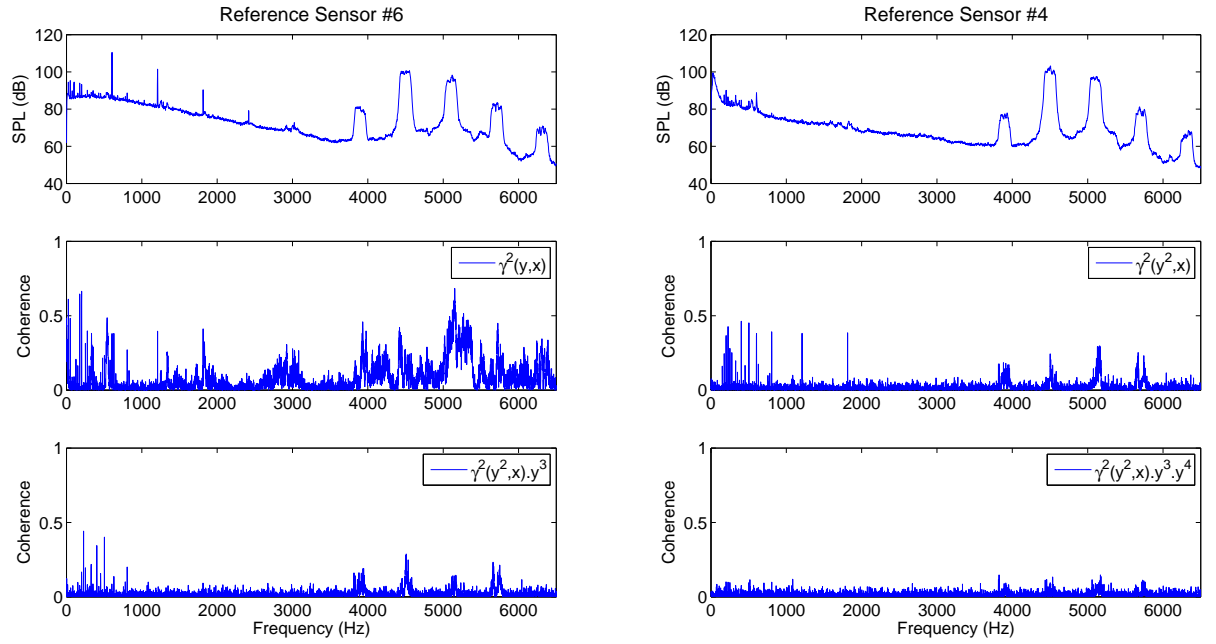

Figure 22. Enhanced non-linear coherence techniques applied to the data of figure 21. Shown is a close-up of the autospectra, the ordinary coherence and also different stages of non-linear component extraction

cut-off" frequency, or the frequency below which there is no sum and difference scattering to be found. Also posited was that, in addition to the sum and difference scattering (or frequency scattering) of the narrowband noise, was that the modal content of the narrowband noise would be scattered also on a modal basis. That is to say, the modal energy is scattered into other frequency ranges with differing modal content. Therefore, depending on the modal content of the initial signal, the modes scattered into the lower difference frequencies, for example, would only propagate in the duct if they were scattered into frequencies above their respective cut-on frequencies. As the experimental work to date: figures 14 and 23; has seen the difference frequencies only, cut-off with decreasing centre frequency, this hypothesis has been supported with experimental evidence. The work of Hanson ${ }^{21}$ characterises mode and frequency scattering effects in 2-D rotors and stators and develops useful modal scattering rules for tones. Of interest here is the modal content of the scattered energy. Eqt. (14) gives expressions for the sum and difference frequencies between an incident frequency $T$ and a blade pass frequency and harmonics. In addition, Eqt. (15) gives the modal content $m^{*}$ of the scattered energy at frequencies of order $h$ given a modal content $m$ of the original tone $T$. The analysis of Hanson supports the hypothesis above. This expression given inn Eqt. (15) needs to be 
verified on this data by performing a modal decomposition on the original tone or narrowband frequencies and subsequently on the scattered energy. However, experiments to data have not resulted in scattered energy in frequency ranges that can be analysed by the microphone arrays in the rig. That is to say, the modal orders are higher than than can be decomposed by the number of microphones present in the arrays. Further tests need to be performed in order to validate the hypothesis.

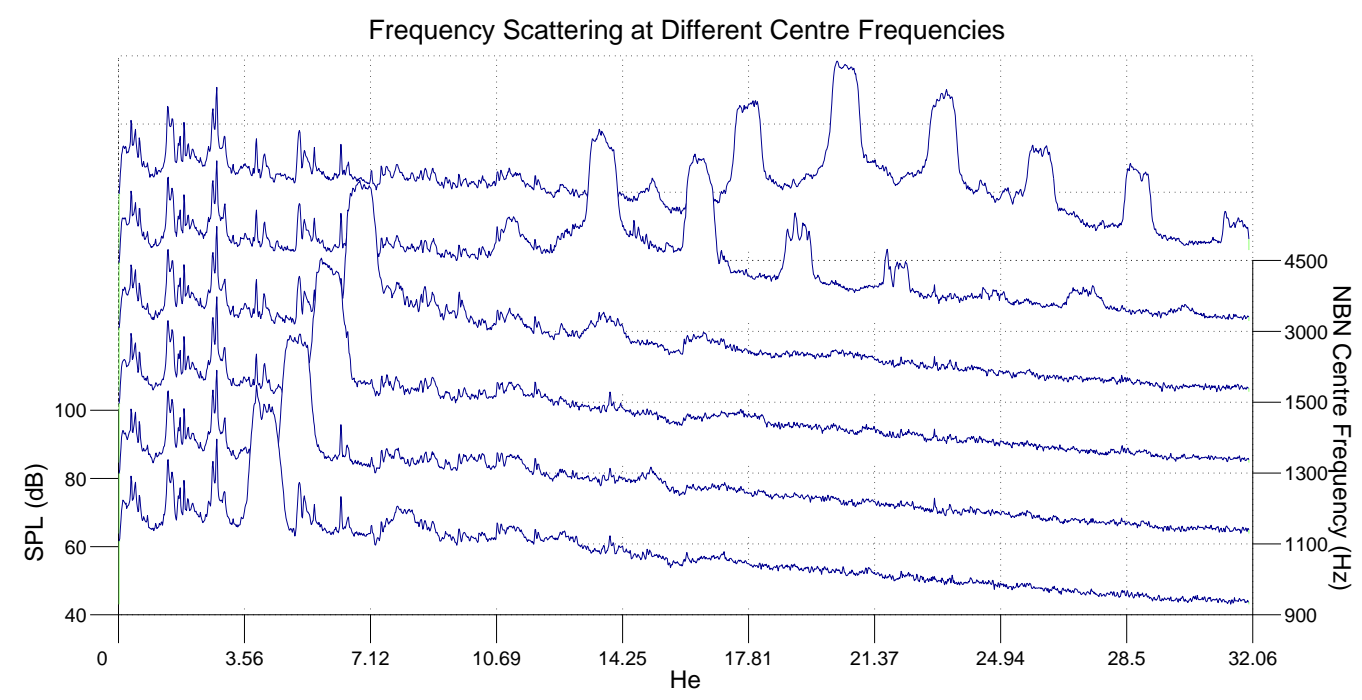

Figure 23. Waterfall plot for a single microphone location as a function of narrowband noise centre frequency. The rotor rpm remains fixed for each test.

$$
\begin{aligned}
f^{*} & =T-(h * B P F) \quad \text { where } \quad h=\cdots-2,-1,0,+1,+2 \ldots \\
m^{*} & =m-(h * B)
\end{aligned}
$$

\section{Conclusions}

In this paper, the ability for coherence based noise source identification techniques to identify core noise in aero-engines was discussed. An experimental rig was designed and built to gain a fundamental physical understanding of the propagation of noise through a rotor/stator set-up. Experiments performed on the rig allowed it to be shown experimentally that acoustic energy in a duct at a certain frequency may interact with rotor/stator noise at a different frequency to scatter energy to a third frequency which is a sum of the two. The case where broad band or narrow band noise, such as may originate from a combustor, as opposed to an upstream rotor, interacts with a rotor-stator pair, (e.g. turbine noise), producing noise at sum and difference frequencies was explored in this paper. An experimental technique was developed which enables the non-linear interaction between the propagated sound source with the vane-axial fan to be detected and identified when present. The technique was extended to allow the linear and non-linear acoustic contributions to be separated.

The capacity to identify non-linear interaction is important as

- Energy in certain frequency ranges will not be the result of linear superposition of known noise sources.

- Energy in certain frequency ranges will change when sound sources producing noise at different frequencies are altered. This makes liner design difficult.

- Reducing a sound source will reduce noise in additional frequency ranges to the ones associated with the sound source.

- Addressing the non-linear interaction mechanism could be more effective in reducing noise than trying to reduce the resulting noise with a liner, for example. 
Experiments to date suggest a "scattering cut-off" frequency, or a frequency below which sum and difference scattering will not occur due to the scattered modes being cut-off. Further tests will focuss on developing a relationship between the scattered narrowband noise and the modal content of the acoustic energy incident on the rotor.

\section{Acknowledgements}

This work was partly supported by the Seventh Framework Programme TEENI project which is funded under EU Commission grant agreement 212367

\section{References}

${ }^{1}$ Karchmer, A. M. and Reshotko, M., "Core noise source diagnostics on a turbofan engine using correlation and coherence techniques," Tech. Rep. TM X-73535, NASA, 1976.

${ }^{2}$ Karchmer, A. M., Reshotko, M., and Montegani, F. J., "Measurement of far field combustion noise from a turbofan engine using coherence function," AIAA 4th Aeroacoustics Conference, No. AIAA-77-1277, Atlanta, Georgia, October 3-5 1977.

${ }^{3}$ Reshotko, M. and Karchmer, A. M., "Core noise measurements from a small general aviation turbofan engine," Tech. Rep. TM81610, NASA, 1980.

${ }^{4}$ Halvorsen, W. G. and Bendat, J. S., "Noise Source Identification Using Coherent Output Power Spectra," Sound and Vibration, Vol. 9, No. 8, 1975, pp. 15, 18-24

${ }^{5}$ Karchmer, A. M., "Conditioned pressure spectra and coherence measurements in the core of a turbofan engine," Tech. Rep. TM82688, NASA, 1981, AIAA Paper 81-2052.

${ }^{6}$ Shivashankara, B. N., "High bypass ratio engine noise component separation by coherence technique," AIAA 7th Aeroacoustics Conference, No. AIAA-81-2054, Palo Alto, October 5-6 1981.

${ }^{7}$ Shivashankara, B. N., "High bypass ratio engine noise component separation by coherence technique," Journal of Aircraft, Vol. 23, No. 10, 1986, pp. 763-767.

${ }^{8}$ Chung, J. Y., "Rejection of flow noise using a coherence function method," J. Acoust. Soc. Am., Vol. 62, No. 2, 1977, pp. 388-395.

${ }^{9} \mathrm{Hsu}$, J. S. and Ahuja, K. K., "A coherence-based technique to separate ejector internal mixing noise from farfield measurements," AIAA/CEAS 4th Aeroacoustics Conference, No. AIAA-98-2296, June 2-4 1998.

${ }^{10}$ Minami, T. and Ahuja, K. K., "Five-microphone method for separating two different correlated noise sources from farfield measurements contaminated by extraneous noise," AIAA/CEAS 9th Aeroacoustics Conference, No. AIAA-03-3261, South Carolina, May 12-14 2003.

${ }^{11}$ Nance, D. K., Separating Contributions of Small-Scale Turbulence, Large-Scale Turbulence, and Core Noise From FarField Exhaust Noise Measurement, Ph.D. thesis, Georgia Institute of Technology, 2007.

${ }^{12}$ Bennett, G. J. and Fitzpatrick, J. A., "A comparison of coherence based acoustic source identification techniques," 12th International congress on sound and vibration, No. 950, Lisbon, Portugal, 11-14 July 2005.

${ }^{13}$ Bennett, G. J. and Fitzpatrick, J. A., "Noise-source identification for ducted fan systems," AIAA Journal, Vol. 46, No. 7, 2008, pp. 1663-1674.

${ }^{14}$ Cumpsty, N. A., "Sum and difference tones from turbomachines," Journal of Sound and Vibration, Vol. 32, No. 3, 1974, pp. 383-386.

${ }^{15}$ Holste, F. and Neise, W., "Noise Source Identification in a Propfan Model by Means of Acoustical Near Field Measurements," Journal of Sound and Vibration, Vol. 203, No. 4, 1997, pp. 641-665.

${ }^{16}$ Enghardt, L., Tapken, U., Neise, W., Kennepohl, F., and Heinig, K., "Turbine Blade/Vane Interaction noise: acoustic mode analysis using in-duct sensor rakes," 7th AIAA/CEAS Conference on Aeroacoustics., No. AIAA-2001-2153, Maastrict, The Netherlands., May 2001.

${ }^{17}$ Nallasamy, M., Hixon, R., Sawyer, S., Dyson, R., and Koch, L., "A Time Domain Analysis of Gust-Cascade Interaction Noise," 9th AIAA/CEAS Aeroacoustics Conference and Exhibit, No. AIAA-2003-3134, Hilton Head, South Carolina, May 12-14 2003.

${ }^{18}$ Bendat, J. S. and Piersol, A. G., Random Data: Analysis and Measurement Procedures, John Wiley \& Sons, 1986.

${ }^{19}$ Esmonde, H., Fitzpatrick, J. A., Rice, H. J., and Axisa, F., "Modeling and Identification of Nonlinear Squeeze Film Dynamics," Journal of Fluids and Structures, Vol. 6, No. 2, 1992, pp. 223-248.

${ }^{20}$ Bennett, G. J., Noise Source Identification For Ducted Fans, Ph.D. thesis, Trinity College Dublin, 2006.

${ }^{21}$ Hanson, D. B., "Acousic reflection and transmission of rotors and stators including mode and frequency scattering." 3rd AIAA/CEAS Aeroacoustics Conference, No. AIAA 1997-1610, Atlanta, Georgia, May 12-14 1997. 